INPLASY

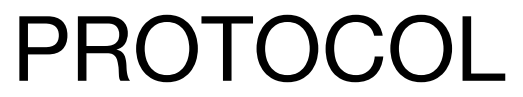

To cite: Chen et al. PED Versus MEDL for the Treatment of Lumbar Spinal Stenosis : Systemic Review and MetaAnalysis. Inplasy protocol 202040188. doi:

10.37766/inplasy2020.4.0188

Received: 26 April 2020

Published: 26 April 2020

Corresponding author: Chen Xin

609131443@qq.com

Author Affiliation:

The first affiliated Hospital of Xiamen University

Support: No

Review Stage at time of this submission: Formal screening of search results against eligibility criteria.

Conflicts of interest:

The authors declare that the article content was composed in the absence of any commercial or financial relationships that could be construed as a potential conflict of interest.

\section{PED Versus MEDL for the Treatment of Lumbar Spinal Stenosis : Systemic Review and Meta-Analysis}

Chen, X; Wang, $\mathrm{ZT}^{2}$.

Review question / Objective: To compare the efficacy and safety of percutaneous endoscopic decompression (PED) and traditional micro endoscopic decompression laminectomy (MEDL) in the treatment of lumbar spinal stenosis (LSS).

Condition being studied: Lumbar spinal stenosis (LSS) is a degenerative condition in which changes in the discs, ligamentum flavum, and facet joints with aging cause narrowing of the spaces around the neurovascular structures of the spine.

Information sources: Retrieval of PubMed, Web of Science, The Cochrane Library, Embase, Chinese journal full-text database, viper database and wanfang database by computer.

INPLASY registration number: This protocol was registered with the International Platform of Registered Systematic Review and Meta-Analysis Protocols (INPLASY) on 26 April 2020 and was last updated on 26 April 2020 (registration number INPLASY202040188).

\section{INTRODUCTION}

Review question / Objective: To compare the efficacy and safety of percutaneous endoscopic decompression (PED) and traditional micro endoscopic decompression laminectomy (MEDL) in the treatment of lumbar spinal stenosis (LSS).

Condition being studied: Lumbar spinal stenosis (LSS) is a degenerative condition in which changes in the discs, ligamentum 
flavum, and facet joints with aging cause narrowing of the spaces around the neurovascular structures of the spine.

\section{METHODS}

Participant or population: Patients who were clinically diagnosed with lumbar spinal stenosis and required surgical treatment, and had not received any surgical intervention before that; There is no limitation on age, sex, race or nationality.

Intervention: Percutaneous endoscopic decompression (PED).

Comparator: Micro endoscopic decompression (MEDL).

Study designs to be included: Randomized controlled trial, case-control trial.

Eligibility criteria: This meta-analysis was carried out in reference to the Cochrane Library's official collaboration.

Information sources: Retrieval of PubMed, Web of Science, The Cochrane Library, Embase, Chinese journal full-text database, viper database and wanfang database by computer.

Main outcome(s): A total of 8 references were included in this study, including 4 randomized controlled studies and 4 nonrandomized controlled studies .A total of 804 patients were involved in the study, and 714 patients were obtained at the last follow-up, with a follow-up rate of $\mathbf{8 8 . 8 \%}$.

Quality assessment / Risk of bias analysis: The quality of 4 randomized controlled studies was evaluated according to the Cochrane collaboration network "risk of bias evaluation criteria", and the quality of 4 non-randomized case control studies was evaluated according to the Cochrane collaboration "newcastal-ottawa Scale (NOS)" evaluation criteria.

Strategy of data synthesis: This metaanalysis used the Review Manager 5.3 software provided by the Cochrane collaboration for statistical analysis.
Subgroup analysis: Different follow-up time and different disease types may be divided into different subgroups for analysis.

Sensibility analysis: The sensitivity analysis will mainly adopt the method of "literature culling one by one".

Country(ies) involved: China, South Korea, Germany.

Keywords: lumbar spinal stenosis; minimally invasive; spinal endoscopy; Discectomy ; Meta-analysis.

Contributions of each author:

Author 1 - Chen Xin - Author 1 drafted the manuscript.

Author 2 - Wang Zhongtang - The author provided statistical expertise. 